\title{
Nanorobotics System Simulation in 3D Workspaces with Low Reynolds Number
}

\author{
Adriano Cavalcanti Tad Hogg Bijan Shirinzadeh \\ CAN Center for Automation in Nanobiotech \\ Sao Paulo, SP 01540, Brazil \\ email: adrianocavalcanti@canbiotechnems.com \\ HP Labs \\ Palo Alto, CA 94304, USA \\ email: tad_hogg@hp.com \\ Robotics and Mechatronics Research Lab., Dept. of Mechanical Eng., Monash University \\ Clayton, Melbourne, VIC 3800, Australia \\ email: bijan.shirinzadeh@eng.monash.edu.au
}

\begin{abstract}
:
We present a computational approach to enable the development of nanorobots operating in a fluid environment relevant for medical applications. Unlike the case of larger robots, the dominant forces in this environment arise from viscosity of low Reynolds number fluid flow and Brownian motion and such parameters are described throughout the paper. Hence, this paper describes a practical simulator that allows fast design methodology comparing various control algorithms for nanorobots and their suitability for different tasks. The simulator includes obstacles and identifiable targets, thereby providing a suitable environment for a typical nanorobot task: maintaining desired chemical concentrations near specific target areas.
\end{abstract}

Keywords: Biomedical computing, mechatronics, mobile robots, nanotechnology, nanomedicine, virtual reality.

\section{InTRODUCTION}

The development of nanoscale robots ("nanorobots") presents difficult fabrication and control challenges [6]. Such devices will operate in microenvironments whose physical properties differ from those encountered by conventional robots. Particularly interesting microenvironments are those involved in nanomedicine applications [13] where the robots operate inside the body to provide significant new capabilities for diagnosis and treatment of disease. In our work we demonstrate a possible design approach to develop control algorithms using the software Nanorobot Control Design (NCD) [4]. The use of 3D visualization is a useful and powerful tool for purpose of designing devices at nanoscale [10]. The focus is on a typical task for such robots: locating specific regions in the environment and maintaining chemical concentrations at desired levels near them [7]. The simulator includes for the organ-inlets thermal and chemical signals which may be detected by the nanorobots' sensors as described in the paper.

Initial uses of nanorobots are expected to enable broad advances in the health care sector [5], [22], [29], [28], [35], [41]. Through the use of nanotechnology techniques [16], genetics advances [39], [44], and biomolecular computing [1], biological nanorobots can be applied in specific cases [24]. For example, in microbiology engineering the construction of digital circuits in living cells has been demonstrated [43]. A standardized set of biological building blocks designed for logic operations inside a cell is being developed successfully [30]. Bacteria have been used as physical system components [23], and radio remote control of biological processes has been demonstrated experimentally [18]. Following similar proposals on nanorobots being injected inside living cells and controlled for biomedical purposes [13], it is expected that the first artificial biological nanorobots to become available in the next five years [12], [27]. Rigid materials based on positional mechanosynthesis are also expected to help in manufacturing inorganic devices to assembly nanorobots [36], [32].

\section{Nanorobot Feasibility}

The proposed nanorobot prototyping methdology must be equipped with the necessary devices for monitoring the most important aspects of its operational workspace which is dominated by low Reynolds number. Depending on the case, the temperature, concentration of chemicals in the blood, and electromagnetic signatures are some of relevant parameters when monitoring human body to detect some diseases [5]. Inside the human body the vessels range from $3 \mathrm{~cm}$ to $6 \mu \mathrm{m}$ (microns) in diameter. More recently a first mobile robot was built that measures $60 \mu \mathrm{m}$ by $250 \mu \mathrm{m}$ [9]. At this scale we can begin to reckon in the coming years robot sizes decreasing rapidly to $50 \mu \mathrm{m}$, and even smaller. This downscaling of electronic devices will continue, according to the Semiconductor Industry Association's roadmap. By 2016, high-performance ICs will contain more than 8.8 billion transistors in a $280 \mathrm{~mm}^{2}$ area - more than 25 times as many as on today's chips built with $130 \mathrm{~nm}$ (nanometers) feature sizes [16]. 


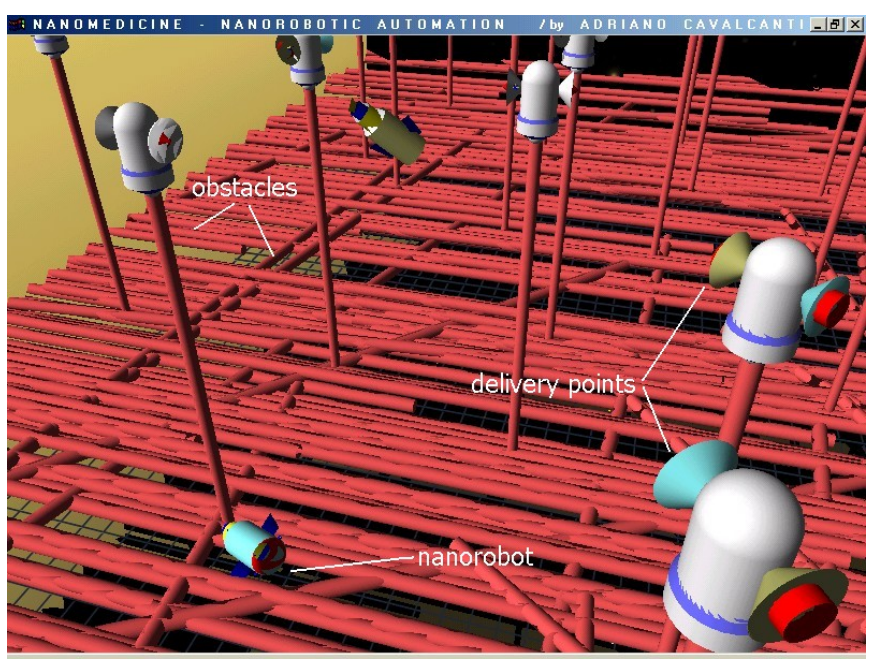

Fig. 1. Virtual environment, top camera view.

The application of new materials has demonstrated a large range of possibilities for use in manufacturing better sensors and actuators with nanoscale sizes [40], [42]. Those developments allied with 3D computational simulation may facilitate the manufacturing design of nanorobots with integrated embedded nanoelectronics and circuits [26]. The CMOS industry may thrive successfully on the pathway for the assembly of nanorobots, where the joint use of nanophotonic and nanotubes may even accelerate further the actual levels of resolution ranging from $248 \mathrm{~nm}$ to $157 \mathrm{~nm}$ devices [3]. The use of yet well established techniques such as SoC (System on Chip) and Lithography, VHDL (Verification Hardware Description Language) and 3D Simulation, allied with recent nanotechnology advances, such as mesoscopic nanowires, may contribute together as a path-way to validate and help the implementation of high complex VLSI featuring functionality with exceptional performance under nanoscale sizes [40], [14]. Thus, it is quite natural to expect the first nanorobots with embedded nanodevices to appear in the next 10 years [38].

\section{Control Problems}

Research efforts have recently been directed towards molecular manufacturing [25]. While the development of nanomedical devices is advancing progressively [8], [33], further investigation on nanorobots for application in medicine may enable significant insights on various aspects such as their operation, sensor, and actuator designs. We consider nanorobots that can move with six-degrees-of-freedom, i.e., arbitrary translation and rotation, and have adequate power resources. We studied a prototypical task for nanorobots in a medical application: moving through a fluid-filled vessel to locate target regions with identifiable characteristics and maintaining chemical concentrations at desired levels near them. The simulator allows examining a variety of control algorithms. A simple example is based on chemotaxis in

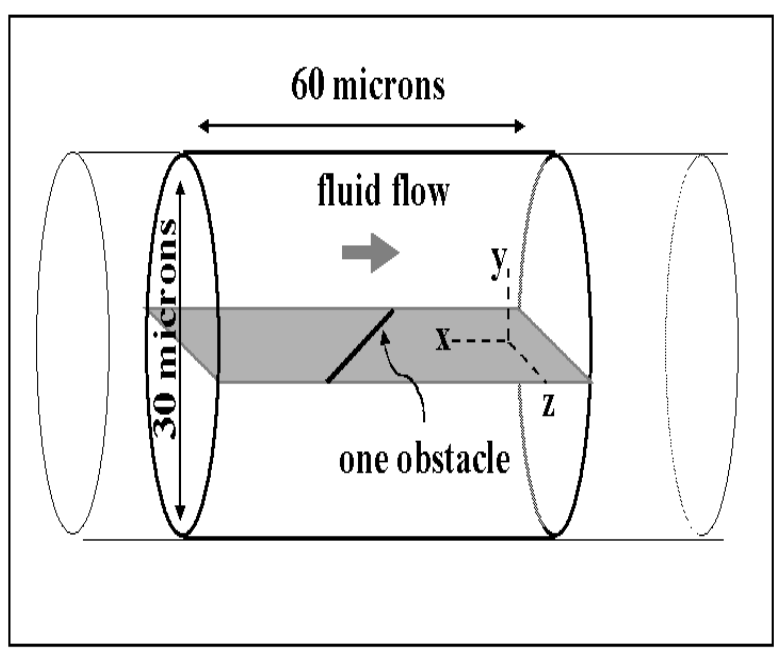

Fig. 2. Schematic illustration of the simulated workspace as a section of a longer pipe. The $\mathrm{x}-\mathrm{z}$ plane contains the obstacles.

bacteria to move along chemical gradients: combining motion with random changes in direction with a frequency based on observed chemical concentrations [2]. The simulator does allow multiple robots to operate independently. The nanorobot attempts to detect and locate any organ-inlet demanding nutrient injection in a period of time for the simulated dynamic scenery (Figure 1).

\section{TASK ENVIronment}

This section describes the task environment used in our simulator. For definiteness, we give specific dimensions to the objects in the environment, though the simulator could also operate with different sizes. Figure 2 schematically shows the simulated microenvironment consists of a long fluid-filled pipe, with diameter of some tens of microns and length much larger than other object sizes, allowing us to neglect effects on the fluid from the ends of the pipe. We take the fluid to have the density and viscosity of plasma. The simulation focus is on the workspace, i.e., the interior of a small section of the overall pipe length. This workspace has a volume of about $40,000 \mu \mathrm{m}^{3}$. We introduce a coordinate system for the simulator with the $\mathrm{x}$ axis along the long axis of the pipe as shown in Figure 2.

\subsection{Components of the Environment}

The 3D environment contains nanorobots, obstacles, biomolecules and specific target regions (see Figure 3). The target regions or delivery points (organ-inlets) are closed when the chemical concentrations are near the desired levels. Otherwise, it will open diffusing chemical and thermal signals to show the required injection of assembled nutrients. A key choice in chemical signalling is the measurement time and detection threshold at which the signal is considered to be received [15]. Due to background concentration, some detection occurs even without the target signal. As a guide for the choice of threshold, we use the diffusive capture rate $\alpha$ for a sphere of radius $R$ in a region with concentration as:

$$
C: \alpha=4 \pi D R C
$$




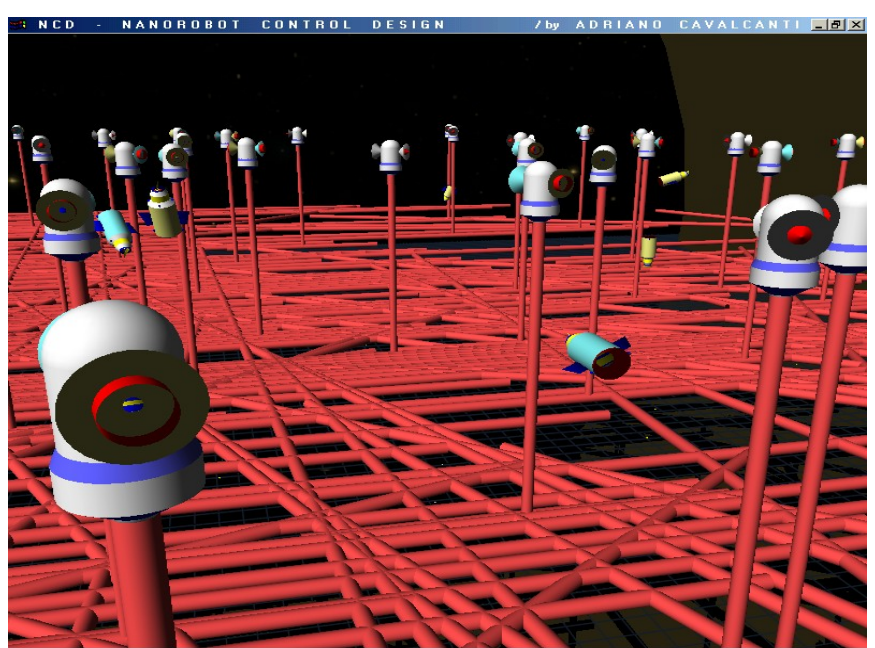

Fig. 3. At second floor nanorobots search for organ-inlets demanding nutrient injection; the biomolecules are in the workspace at first floor.

where the concentration for other shapes such as cylinders are about the same [37], [2]. All moving objects (i.e., the nanorobots and biomolecules) in the workspace have neutral buoyancy. Each nanorobot is a cylinder $600 \mathrm{~nm}$ in length and with a diameter of $200 \mathrm{~nm}$.

The environment includes a rigid, fixed structure of obstacles created as follows. We start with a specified number of 200 points, randomly placed in the x,z-plane that cuts the pipe in half, as illustrated in Figure 3. For each of these points, we associate an orientation, selected randomly from 0,45 or 90 degrees of rotation about the y-axis. From each point, we then grow a cylinder, $100 \mathrm{~nm}$ in diameter, directed in the orientation associated with the point. The cylinder grows until it meets the boundary of the pipe or for $10 \mu \mathrm{m}$ from the original point. We also grow the cylinder in the opposite direction from the starting point, again for $10 \mu \mathrm{m}$ or until it reaches the boundary. Thus these cylinders are $20 \mu \mathrm{m}$ in length unless shortened due to reaching the pipe's boundary, at which point we take the cylinder to be rigidly attached to the boundary. Whenever two such cylinders intersect, we consider them to be joined together into a single solid structure, i.e., there will be no freefloating cylinders. This construction creates a random network of obstacles in the plane bisecting the pipe (Figure 5). Typically we chose a number of obstacles in the workspace, which is more than sufficient to ensure no free-floating cylinders. The nanorobot cannot move through gaps between obstacles smaller than the robot's size.

The environment includes various target regions as organinlets (e.g., to represent target locations or delivery points for medical applications). The environment includes also spheres with $10 \mathrm{~nm}$ diameter (the size of a typical protein) to represent various bio-molecules that the nanorobots can use. These spheres move with the fluid, and follow the laminar flow [34], [11] considered additional Brownian motions. We take their concentration to be about 200 biomolecules with the nanorobots in the workspace at any one time.

\subsection{Fluid Motion}

We take the fluid in the pipe to move with velocity $\sim 1 \mathrm{~mm} / \mathrm{sec}$, as is typical of flow in small blood vessels [13], [21]. Even at our size scale, the distances are large compared to the flowing molecules so the fluid is still described by the classical continuum equations [10], [31]. We treat the fluid as incompressible so the fluid velocity $v$ satisfies the continuity condition $\nabla \cdot v=0$ and the Navier-Stokes equation:

$$
\frac{\partial v}{\partial t}+(v \cdot \nabla) v=f-\frac{1}{\rho} \nabla P+\frac{\eta}{\rho} \nabla^{2} v
$$

where $\eta$ is the fluid's viscosity, $\rho$ its density, $P$ is the pressure and $f$ is the external force, per unit mass, imposed on the fluid. The three components of the Navier-Stokes equation and the continuity condition give the equation for the components of the velocity and the pressure. For slow motions this equation simplifies considerably. Specifically, in contrast to the conventional and large-scale robots, the nanorobot's world is dominated by viscosity while inertial and gravitational forces are negligible [10], [13], [37]. The Reynolds number, defined as

$$
\operatorname{Re}=L \rho v / \eta
$$

for objects of size $L$ with velocity $v$, characterizes this behavior by giving the ratio of inertial to viscous forces.

\subsection{Object Motion}

Our environment contains two types of moving 3D objects: the nanorobots and the small spheres representing biomolecules. These objects are subject to both deterministic and random forces. The deterministic forces arise from the fluid motion and, in the case of the nanorobots, from their powered locomotion. Brownian motion gives additional random displacements. The nanorobots can move in respect to the fluid with velocities up to a few millimeters per second. The low Reynolds number flow means inertial forces on the objects are insignificant. Specifically, the inertial force on the object of size $L$ moving with velocity $v$ with respect to the fluid is of order $F_{\text {inertial }} \cong \rho v^{2} L^{2}$ and the viscous drag force is of order $F_{\text {viscous }} \cong \eta v L$. Two main forces act on a nanorobot while it is not in contact with other objects. First is the force $F_{r}$ produced by the robot itself, which we take to be directed along the axis of the cylinder. Second is the drag from the fluid given by:

$$
F_{\text {drag }}=-A_{\text {drag }} \eta L v
$$

where $v$ is the velocity vector of the nanorobot with respect to the fluid, $v=v_{\text {robot }}-v_{\text {fluid }}$. The quantity $A_{d r a g}$ is a geometric factor depending on the orientation of the robot with respect to the fluid and is typically of order 1, e.g., for a sphere of diameter $L, A_{d r a g}$ is $3 \pi$ when no other objects are nearby.

For other situations, $A_{\text {drag }}$ has roughly the same magnitude, but the exact value must be determined numerically. To see 
how this is done, consider a small area $d A$ on the surface of an object, treated as a vector oriented perpendicular to the surface. The fluid imposes a force vector $-T d A$ on that area, where $T$ is a matrix representing the stress tensor for the fluid motion at the surface of the object. For incompressible fluids, its components are expressed by

$$
T_{k, l}=P \delta_{k, l}-\eta\left(\frac{\partial v_{k}}{\partial x_{l}}+\frac{\partial v_{l}}{\partial x k}\right) .
$$

where is $\delta_{k, l}=1$ when $k=l$ and is 0 otherwise.

In general, the velocity gradient and pressure vary over the surface of the object. The total drag force requires integrating the force on each part of the object. The difference in forces around the object can also give rise to a torque, causing the object to rotate as it moves through the fluid.

\section{Nanorobot Capabilities}

In this section, we describe the nanorobot capabilities for moving, sensing and manipulating.

\subsection{Locomotion}

The robots have a propulsion mechanism to move through the fluid. A variety of mechanisms are possible [37], [13]. As one example, the robots have double propellers that operates at four levels: off (no additional force), normal, slow (50\% of normal) and fast (150\% of normal). Since applied forces rapidly produce a terminal velocity through the fluid, in the simulation of the locomotion mechanism produce a given velocity $\mathrm{v}_{\text {force }}$ with respect to the fluid according to Eq. (6). Specifically, we take the normal, slow and fast propeller levels to produce velocities of $2 \mathrm{~mm} / \mathrm{sec}, 1 \mathrm{~mm} / \mathrm{sec}$ and $3 \mathrm{~mm} / \mathrm{sec}$, respectively. As described in Eq. (6), these speeds correspond to forces of order $10^{3} \mathrm{fN}$.

$$
v_{\text {robot }}=v_{\text {fluid }}+v_{\text {force }}
$$

with $\mathrm{v}_{\text {force }}$ directed along the axis of the nanorobot and with one of these three magnitudes.

\subsection{Sensing}

We simulate the nanorobot with sensory capabilities allowing it to detect and identify the nearby large objects in its environment, as well as the target regions for its task. A variety of sensors are possible [13]. For instance, chemical detection can be very selective, e.g., for identifying types of cells by markers on their surfaces. Acoustic sensing is another possibility. In the present study, the nanorobts don't need to communicate with each other. But when required active communication among nanorobots, sonar communication frequencies may reache up to $20 \mu \mathrm{W} @ 8 \mathrm{~Hz}$ as ressonance rates and 3V supply [20], [19]. The nanorobot includes external sensors to inform it of collisions and to identify when it has encountered a chemical signal or abrupt changes of temperature for targeted areas. It is well known that there is a significant temperature rise every time there is some abnormality in the human body [5]. The temperature difference
TABLE I

PARAMETERS

Chemical signal

\begin{tabular}{lc} 
production rate & $Q=10^{4}$ molecule $/ \mathrm{s}$ \\
diffusion coefficient & $D=100 \mu \mathrm{m}^{2} / \mathrm{s}$ \\
background concentration & $6 \times 10^{-3}$ molecule $/(\mu \mathrm{m})^{3}$ \\
\hline \multicolumn{1}{c}{ Parameter } & Nominal value \\
\hline average fluid velocity & $v=1000 \mu \mathrm{m} / \mathrm{s}$ \\
vessel diameter & $d=10 \mu \mathrm{m}, 20 \mu \mathrm{m}, 40 \mu \mathrm{m}$ \\
workspace length & $L=60 \mu \mathrm{m}$ \\
density of nanorobots & $2 \times 10^{-4} \mathrm{robot} /(\mu \mathrm{m})^{3}$ \\
\hline
\end{tabular}

at the site of some lesion from the core temperature can reach up to $\sim 2^{\circ} \mathrm{C}$ [28]. Hence, in order to simulate the nanorobot intervention and interaction with the workspace, we used different organ-inlets as delivery targets, where depending on their nutrient demand, they will be emitting chemical and thermal signals. These environment changes will be used to guide the nanorobots, as described further.

\subsection{Manipulation}

In our study, the nanorobot's task involves collecting various molecules, assembling them and delivering the results to specific target regions in the environment. For collecting molecules, the nanorobot uses molecular sorting rotors [10] to capture and deliver molecules and a general manipulator, such as an assembler performing specific chemical reactions [13]. Normally some proteins can be converted into glucose. In our study the nanorobots are comprised internally by telescoping manipulators for the assembly of proteins into nutrients [10]. The nanorobots must after processing biomolecules into nutrients, use control strategies involving movement around the environment to identify and reach the organ-inlets requiring delivery of nutrients.

\section{Simulator Implementation and Results}

The simulator maintains a list of positions and orientations of all objects in the task environment, including the nanorobots. This list maintains all information relevant for the nanorobot tasks. It also introduces new biomolecules with the fluid as it enters the environment. The simulator consists of several modules, that simulate physical behaviors, determine sensory information for each robot, run the nanorobot control programs to determine their actions, provide a visual display of the environment, and record the history of robot behaviors for later analysis. The NCD simulator allows arbitrary control programs for the nanorobots, which determine their actions based on their sensor information. For biomedical applications, the fluid velocity should vary in time and space as objects, such as cells, move through the vessel. The obstacles found in the bloodstream include floating cells that can deform and attach to the boundary, giving a more complex dynamic environment for the nanorobot navigation as considered in another work [5], [17]. 


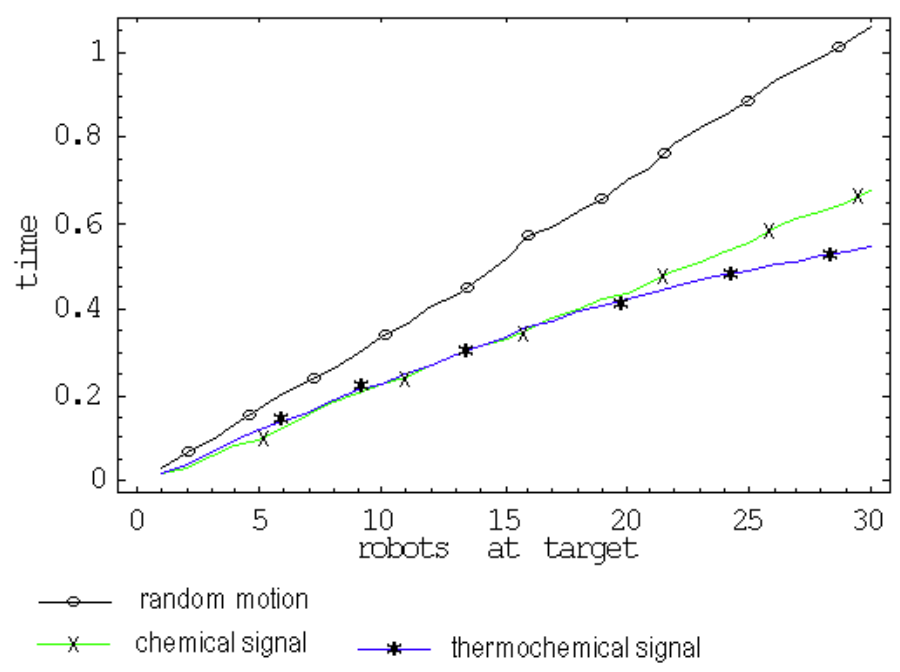

Fig. 4. Time for the nanorobots reach the targets with random motion, chemical signals, and thermochemical signal in a $20 \mu \mathrm{m}$ diameter vessel.

\subsection{Numerical Results}

We simulated distinct cases to validate our study given the Eq. (1) and parameters on table I for diffusing signals. In our study there exist 30 nanorobots to perform similar tasks on detecting and acting upon targets demanding nutrients injections (Figure 3). It is also performed the comparison for different cases in terms of monitoring how effective could the nanorobot behaviors be in the workspace, sensing and detecting targets in the workspace using different parameters (Figure 4). For 20 micron vessel, this shows average time to have $1,2, \ldots, 30$ nanorobots at the organ-inlets with behavior guided for random motion (case 1), by sensing chemical signal (case 2), or even navigation based on target with thermochemical signals (case 3 ). Note how the chemical allied with thermal signals allows nanorobots to find their targets faster. We can see the thermal signal can aid a long term improvement for nanorobots to increase the speed to detect and reach the right target demanding nutrient injections, so the curve in the plot bends downward, while the other two shows longer time to detect and reach the organ-inlets demanding nutrients.

For the case study with the 40 micron vessel the numerical results can be seen in Figure 5. Here the chemical signal in comparison with the thermochemical signal does not differ. In the plot the chemical and thermochemical signals are quite similar for a larger vessel, thus both lines overlap together in the graph showing a similar behavior for the nanorobots. Apparently, the larger vessel is too large for the extra thermal signal to make a difference in terms of nanorobot sensors to substantially improve its performance. It takes also a longer time for nanorobots to find the target detecting the signal. Thus, thermochemical signals require more time to diffuse far enough to make a difference for a larger vessel. However, the performance is still clearly better than using simple random motion for nanorobot control.

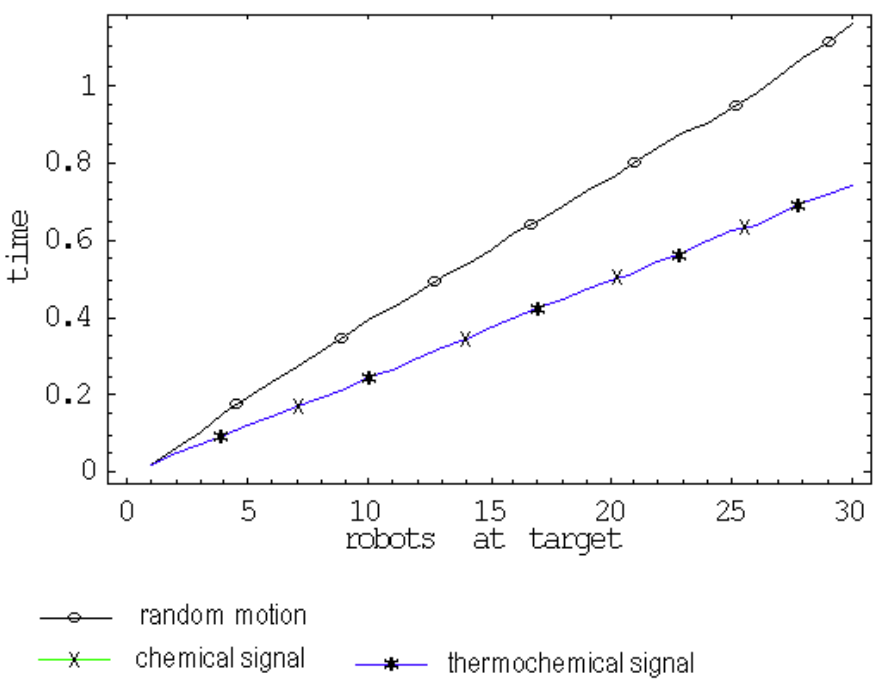

Fig. 5. Nanorobot behavior for random motion, chemical detection, and thermochemical sensing, for a $40 \mu \mathrm{m}$ diameter vessel; chemical and thermochemical lines overlap together.

Therefore, in low Reynolds number, for smaller vessels the chemical and thermochemical signals have a faster influence on the nanorobot performance, enabling it to better accomplish the assigned tasks on control of nutrient concentrations.

\section{Conclusion}

Processing proteins into nutrients is common and serious problem for people suffering from bulimia or anorexia. The detailed framework we presented, describing successfully the activation and control of nanorobots to monitor nutritional levels, can help to develop control system for nanorobots. It can also be useful to design manufacturing approaches for embedded sensor and actuator. Thus, the system we implemented may enable an insightful preview of upcoming feasible directions to practical implementation of nanorobots, both in system as in hardware.

Some biomedical problems should benefit first from nanorobots due their clear and direct relationship with chemical, electromagnetic, thermal or cellular based therapies and signals. Among those diseases, is worth to mention: cardiovascular problems, different types of cancers, and diabetes. Basically, in these three specific cases, our team is currently carrying out some studies based on clinical data. Our goals with such studies and simulations are quite clear: define effective ways of controlling nanorobots inside the human body; specify how thermal and chemical changes affect the low Reynolds number environments which comprise the human body; and utilizing these results specify system on chip sensors and actuators for assembly and manufacturing nanorobots. In the coming years nanorobots may help relieve people's suffering from a large number of diseases. We are working now in this direction. 


\section{ACKnowledgment}

The authors thank Robert A. Freitas Jr., Lior Rosen, Luiz C. Kretly, and Denny Oetomo, for helpful comments provided on the project of this paper.

\section{REFERENCES}

[1] L. M. Adleman, "On Constructing A Molecular Computer", DNA Based Computers, 1995, http://olymp.wu-wien.ac.at/usr/ai/frisch/local.html .

[2] J. P. Adler, "Chemotaxis in Bacteria", Science 153:708-716 (1966).

[3] W. Bogaerts, R. Baets, P. Dumon, V. Wiaux, S. Beckx, D. Taillaert, B. Luyssaert, J.V. Campenhout, P. Bienstman, D.V. Thourhout, "Nanophotonic Waveguides in Silicon-on-Insulator Fabricated with CMOS Technology", Journal of Lightwave Technology, vol. 23, no. 1, pp. 401-412, Jan. 2005.

[4] A. Cavalcanti, R.A. Freitas Jr., "Nanorobotics Control Design: A Collective Behavior Approach for Medicine", IEEE Transactions on Nanobioscience, Vol. 4, no. 2, pp. 133-140, June 2005.

[5] A. Cavalcanti, L. Rosen, L.C. Kretly, M. Rosenfeld, S. Einav, "Nanorobotics challenges in biomedical applications, design and control," IEEE ICECS International Conference on Electronics, Circuits and Systems, Tel-Aviv, Israel, pp. 447-450, December 2004.

[6] A. Cavalcanti, "Assembly Automation with Evolutionary Nanorobots and Sensor-Based Control applied to Nanomedicine", IEEE Transactions on Nanotechnology, Vol. 2, no. 2, pp. 82-87, June 2003.

[7] P. Couvreur, C. Vauthier, "Nanotechnology: Intelligent design to treat complex disease", Pharmaceutical Research, vol. 23, no. 7, pp. 14171450 Jul. 2006.

[8] A.S.G. Curtis, M. Dalby, N. Gadegaard, "Cell signaling arising from nanotopography: implications for nanomedical devices", Nanomedicine Journal, Future Medicine, vol. 1, no. 1, pp. 67-72, June 2006.

[9] B. Donald, "Dartmouth researchers build world's smallest mobile robot", Dartmouth News, Sep. 2005, www.dartmouth.edu/ news/releases/200 5/09/14.html.

[10] K.E. Drexler, "Nanosystems: molecular machinery, manufacturing, and computation", Wiley \& Sons, 1992.

[11] L.J. Fauci, S. Gueron, “Computational Modelling in Biological Fluid Dynamics", Springer, Berlin, 2001.

[12] R.A. Freitas Jr., R.C. Merkle, "Kinematic Self-Replicating Machines", Landes Bioscience, Georgetown, TX, 2004. http://www.MolecularAssembler.com/KSRM.htm.

[13] R.A. Freitas Jr., "Nanomedicine", Vol. I: Basic Capabilities, Landes Bioscience, 1999, www.nanomedicine.com .

[14] C.K.M. Fung, W.J. Li, "Ultra-low-power Polymer Thin Film Encapsulated Carbon Nanotube Thermal Sensors", IEEE Conf. on Nanotechnology, pp.158-160, Aug. 2004.

[15] A. Galstyan, T. Hogg, K. Lerman, "Modeling and Mathematical Analysis of Swarms of Microscopic Robots", IEEE Swarm Intelligence Symposium, pp. 201-208, Pasadena CA, USA, June 2005.

[16] L. Geppert, "The Amazing Vanishing Transistor Act", Cover story, IEEE Spectrum Magazine, pp. 28-33, October 2002.

[17] Globo, "Network Television Broadcast on Nanorobots for Medicine", 2'36" time, Interview in Portuguese, EPTV Campinas, Rede Globo www.globo.com, January 2005, www.nanorobotdesign.com/video/nanorobots.wmv.

[18] K. Hamad-Schifferli, J.J. Schwartz, A.T. Santos, S. Zhang, J.M. Jacobson, "Remote electronic control of DNA hybridization through inductive coupling to an attached metal nanocrystal antenna," Nature $415 \quad(10$ January 2002), pp. 152-156; http://www.media.mit.edu/molecular/HamadNature.pdf.

[19] R.K. Hansen, P.A. Andersen, "A 3-D underwater acoustic camera properties and applications," In P. Tortoli and L. Masotti, editors, Acoustical Imaging, Plenum Press, pp. 607-611, 1996.

[20] T.K. Horiuchi, R.E. Cummings, "A Time-Series Novelty Detection Chip for Sonar", Int'l J. of Robotics and Automation, ACTA Press, 2004.

[21] G.E. Karniadakis, A. Beskok, "Micro Flows: Fundamentals and Simulation", Springer, New York, 2002.

[22] E. Katz, A. Riklin, V.H. Shabtai, I. Willner, A.F. Bückmann, "Glucose Oxidase Electrodes via Reconstitution of the Apo-Enzyme: Tailoring of Novel Glucose Biosensors", Anal. Chim. Acta., 385, 45-58, 1999.
[23] J.W. Kim, A. Malshe, S. Tung, "Bio-inspired MEMS: A novel microfluidics system actuated by biological cell motors", 2003 Institute of Biological Engineering (IBE) Annual Meeting, Athens, GA, 2003.

[24] S.P. Leary, C.Y. Liu, M.L.I. Apuzzo, "Toward the emergence of nanoneurosurgery: Part III - Nanomedicine: Targeted nanotherapy, nanosurgery, and progress toward the realization of nanoneurosurgery", Neurosurgery, vol. 58, no. 6, pp. 1009-1025 Jun. 2006.

[25] W.J. Li, N. Xi, W.K. Fung, T.S. Wong, "Nanorobotics and Nanomanipulation", Encyclopedia of Nanoscience and Nanotechnology, American Scientific Publishers, vol. 7, no. 15, pp. 351-365, 2004.

[26] W. Liang, W.T. Shay, M.S. Huang, W.J. Tseng, "The Progress of Photonic Sensor Development to Measure the RF Electric and Magnetic Fields", IEEE Asia-Pacific Conf. on Environmental Electromagnetics CEEM' 2003 , Haagzhou, China, November 2003.

[27] J.S. MacNeil, "Nanorobot Pioneer Reveal Status of Simulator, Stem Cell Work," NanoBiotech News, Vol. 2, n. 36, pp. 4-5, September 2004, nanorobotdesign.com/article/nanorobotNanoBiotechNews.pdf.

[28] M.R. McDevitt, D. Ma, L.T. Lai, J. Simon, P. Borchardt, R.K. Frank, K.Wu, V. Pellegrini, M.J. Curcio, M. Miederer, N.H. Bander, D.A. Scheinberg, "Tumor Therapy with Targeted Atomic Nanogenerators," Science 294, 16 November 2001, pp. 1537-1540, Nov. 2001. http://www.sciencemag.org/cgi/content/full/294/5546/1537.

[29] A.J. Menezes, V.J. Kapoor, V.K. Goel, B.D. Cameron, J.Y. Lu, "Within a Nanometer of your Life", Mechanical Eng. Magazine, Aug. 2001, www.memagazine.org/backissues/aug01/features/nmeter/nmeter.html.

[30] MIT Registry of Standard Biological Parts, formerly BioBricks Alpha Data Book, http://parts.syntheticbiology.org.

[31] B.R. Munson, D.F. Young, T.H. Okiishi, "Fundamentals of Fluid Mechanics", John Wiley \& Sons, NY, 1997.

[32] R.J. Narayan, "Pulsed laser deposition of functionally gradient diamondlike carbon-metal nanocomposites", Diamond and Related Materials, vol. 14, no. 8, pp. 1319-1330 Aug. 2005.

[33] R.J. Narayan, P.N. Kumta, C. Sfeir, D.H. Lee, D. Olton, D. Choi, "Nanostructured ceramics in medical devices: Applications and prospects", JOM, vol. 56, no. 10, pp. 38-43 Oct 2004.

[34] D.S. Nichols, "Development of a Free Surface Method Utilizing an Incompressible Multi-Phase Algorithm to Study the Flow About Surface Ships and Underwater Vehicles", PhD Dissertation, Mississippi State University, August 2002.

[35] G.M. Patel, G.C. Patel, R.B. Patel, J.K. Patel, M. Patel, "Nanorobot: A versatile tool in nanomedicine", Journal of Drug Targeting, 14 (2): 63 $67 \mathrm{Feb} 2006$.

[36] J. Peng, R.A. Freitas Jr., R.C. Merkle, "Theoretical Analysis of Diamond Mechanosynthesis. Part I. Stability of C2 Mediated Growth of Nanocrystalline Diamond C(110) Surface," J. Comput. Theor. Nanosci. 1(March 2004), pp. 62-70.

[37] E.M. Purcell, "Life at Low Reynolds Number", American Journal of Physics, 45:3-11 (1977).

[38] V. Sa, Medical Nanorobotics Feasibility, Nanotechnology Now, November 2005, www.nanotech-now.com/Adriano-CavalcantiNovember05.htm.

[39] E. Smalley, "Tools design DNA-nanotube logic," Technology Research News, Vol. 139, n.2, pp. 26-28, September 2004.

[40] N. Srivastava, K. Banerjee, "Performance analysis of carbon nanotube interconnects for VLSI applications", IEEE/ACM ICCAD Int'l Conf. on Computer-Aided Design, pp. 383-390, Nov. 2005.

[41] T. Toth-Fejel, "Agents, assemblers, and ANTS: scheduling assembly with market and biological software mechanisms," Nanotechnology 11, pp. 133-137, 2000.

[42] I.D. Villar, I.R. Matias, F.J. Arregui, R.O. Claus, "ESA-based in-fiber nanocavity for hydrogen-peroxide detection", IEEE Transactions on Nanotechnology, vol. 4, no. 2, pp. 187-193 Mar. 2005.

[43] Y. Yokobayashi, R. Weiss, F.H. Arnold, "Directed evolution of a genetic circuit," Proc. Natl. Acad. Sci. 99, 24 December 2002, pp. 16587-16591, USA, Dec. 2002

[44] M.J. Zhang, C.L. Sabharwal, W. Tao, T.J. Tarn, N. Xi, G. Li, "Interactive DNA sequence and structure design for DNA nanoapplications", IEEE Transactions on Nanobioscience, vol. 3, no. 4, pp. 286-292 Dec. 2004. 